\title{
Study on Applicability of Genotype $\times$ Yield $\times$ Trait $($ GYT) Biplots over Genotype $\times$ Trait (GT) Biplots in Selection of Maize Hybrids across Soil Moisture Regimes
}

\author{
Ashok Singamsetti ${ }^{1}$, J.P. Shahi ${ }^{1}$, P.H. Zaidi ${ }^{2}$, K. Seetharam ${ }^{2}$
}

10.18805/IJARE.A-5850

\begin{abstract}
Background: A large area of the maize production in Indo-Gangetic plains of India exposes alternative and combination of prolonged drought spells and heavy rainfall situation due to uncertainty of monsoon patterns. In such context, breeding for maize hybrids with cross-tolerant to both low and excess soil moisture stresses remain the ultimate alternative.

Methods: Evaluation of 75 maize hybrids planted at Banaras Hindu University, India during the subsequent Rabi and Kharif seasons of 2017 and 2018, respectively. The hybrids were evaluated for acquiring information on inter-trait associations among yield and yield-related traits under different soil moisture regimes. The trials were planted in alpha-lattice design and managed stress was imposed and data recorded.

Result: As our investigation was the evident of weak correlations among the traits studied, genotype $\times$ trait (GT) biplots are not advisable to select/ discard the genotypes under moisture stress conditions. The goodness of fit for GT biplots constructed under studied moisture conditions were almost poor because of fair to moderate correlations among the traits. To accomplish this problem of weak associations, genotype $\times$ yield $\times$ trait (GYT) technique was advisable to perform the precise selection of genotypes under studied environments. The investigation emphasized on accuracy and adequacy in implementing of GYT biplots for characterizing and selecting suitable hybrids.
\end{abstract}

Key words: Correlation, Drought, GT biplots, GYT biplots, Waterlogging

\section{INTRODUCTION}

Approximately, 15 million farmers in India directly engaged in maize cultivation, and 45 million metric tons of maize production was targeted by the year 2022 to meet the nation's demand. But, only $15 \%$ of the cultivated area of maize is under proper irrigation and water availability at crucial growth stages has been a greater challenge to achieve sustainability in maize production (Sah et al. 2020). On other side, the crop grown during the summer-rainy seasons in the Indo-Gangetic plains critically face waterlogging due to contingent flooding and continuous rainfall. In South and South-East Asia alone, around 18\% of the total maize cultivating area is frequently affected by floods (Zaidi et al. 2008). Maize is comparatively more sensitive to moisture stresses such as drought and waterlogging at flowering and knee-high stage, respectively that leads to severe yield losses through poor seed set because of barrenness or reductions in kernels rows and kernels per ear (Bolaños and Edmeades, 1993).

The study of correlations along with variability would assist the plant breeder in improving a character that brings simultaneous improvement in other characters. Al-Naggar et al. (2020) conducted an experiment to evaluate nineteen maize genotypes in two seasons with two irrigation regimes such as well-watered and water stressed at flowering stage. Similarly, field experiments were carried out by Nelimor et al. (2020) over two years on 66 extra-early maize landraces under drought, heat, combined stresses and stress-free
${ }^{1}$ Department of Genetics and Plant Breeding, Institute of Agricultural Sciences, Banaras Hindu University, Varanasi-221 005, Uttar Pradesh, India.

${ }^{2}$ The International Maize and Wheat Improvement Center (CIMMYT), ICRISAT Campus, Hyderabad-502 324, Telangana, India.

Corresponding Author: Ashok Singamsetti, Department of Genetics and Plant Breeding, Institute of Agricultural Sciences, Banaras Hindu University, Varanasi-221 005, Uttar Pradesh, India.

Email: ashoks.setti10@bhu.ac.in

How to cite this article: Singamsetti, A., Shahi, J.P., Zaidi, P.H. and Seetharam, K. (2022). Study on Applicability of Genotype $\times$ Yield $\times$ Trait $(G Y T)$ Biplots over Genotype $\times$ Trait (GT) Biplots in Selection of Maize Hybrids across Soil Moisture Regimes. Indian Journal of Agricultural Research. DOI: 10.18805/IJARe.A-5850.

Submitted: 14-06-2021 Accepted: 06-01-2022 Online: 04-03-20222

conditions. Although, a numerous works reported on genetic variability and correlation studies in maize, but the present experiment was aimed to decipher the effect of soil moisture on associations among yield and other corresponding traits. Dealing with data of several traits from a multi-environment test is a challenge due to presence of unfavourable associations among traits (Yan et al., 2019). Since, nonlinear distribution of data and poor correlations among traits under stress conditions, selection of genotypes based on genotype $\times$ trait (GT) biplots was not accurate. To overcome 
the weak inter trait association, a novel tool of genotype $x$ yield $\times$ trait $(G Y T)$ biplot was proposed to visualize the yieldtrait combinations that increase the goodness of fit for the biplots (Yan and Frégeau-Reid, 2018). Sofi et al. (2021) studied to assess the comparative efficiency of GYT approach over GT approach in genotypic selection on multiple traits in common bean. The objective of the investigation was to estimate the magnitude of genetic variability and interrelationships among grain yield and its attributing traits among 75 maize hybrids under low, optimal and excess soil moisture condition; and construction of GT and GYT biplots for individual moisture condition to visualize the variability and association of traits and yield-trait combinations for the test hybrids.

\section{MATERIALS AND METHODS}

A set of 75 maize hybrids including five checks shown in Table 1 were developed from a CIMMYT's germplasm pool of 600 diverse maize lines majorly sourced from Asian tropical and subtropical regions that crossed with two elite testers viz, CML451 and CL02450 through biparental crossing pattern. These genotypes were evaluated under four environments in two seasons, under three soil moisture regimes such as optimal (OSM), low (LSM) and excess soil moisture (ESM) at Varanasi during the Rabi 2017-18 and Kharif 2018. Test environments E1 and E2 were planted in last week of December in Rabi 2017 under LSM and OSM, respectively whereas E3 and E4 in the first week of July in
Kharif 2018 under OSM and ESM conditions, respectively. The experimental layout was alpha-lattice design $(0,1)$ with two replications. Managed drought stress and waterlogging stress imposed based on the abiotic stress phenotypic manuals by CIMMYT (Zaman-Allah et al., 2016; Zaidi et al., 2016). Manual sowing was done with $4 \mathrm{~m}$ in row length and two in row number and spacing was $60 \times 20 \mathrm{~cm}$. The phenotypic data on days to $50 \%$ anthesis (ANTH), days to $50 \%$ silking (SILK), anthesis-silking interval (ASI), shoot length (SL), chlorophyll content (SPAD), ear length (EL), number of kernels per row (NKPR), test weight (TW) and grain yield per hectare (GY) was recorded. Shelling and moisture percent for each plot were estimated for conversion of fresh weight of ears without husk in to grain yield per hectare (GY, in t/ha) at $15 \%$ moisture.

The data were subjected to ANOVA by general linear mixed model (GLMM). The data subjected to PROCGLM of SAS with the RANDOM statement (SAS, 2009). In the statistical model, replications and incomplete blocks within replication were considered as random, while the genotypes, soil moisture conditions within season was treated as a fixed factor. Soil moisture condition of the crop growing period was nested within 'season' factor. The model followed by Rusinamhodzi et al. 2020 was used in experiment. Magnitude and direction of association among the yield and its component traits were estimated by Pearson's correlation method. To visualize the genetic variability among test hybrids under the three test environments, a principal

Table 1: List of maize genotypes evaluated under different soil moisture conditions

\begin{tabular}{|c|c|c|c|c|c|}
\hline Code & Genotype name & Code & Genotype name & Code & Genotype name \\
\hline 1 & ZH161271 & 26 & ZH161042 & 51 & ZH161478 \\
\hline 2 & $\mathrm{ZH} 161475$ & 27 & ZH161079 & 52 & ZH161456 \\
\hline 3 & ZH161493 & 28 & ZH161076 & 53 & ZH161484 \\
\hline 4 & $\mathrm{ZH} 161464$ & 29 & ZH161039 & 54 & ZH161463 \\
\hline 5 & ZH161409 & 30 & ZH161047 & 55 & ZH15449 \\
\hline 6 & $\mathrm{ZH} 161361$ & 31 & ZH161100 & 56 & ZH161196 \\
\hline 7 & ZH161485 & 32 & $\mathrm{ZH} 161045$ & 57 & ZH161194 \\
\hline 9 & ZH161384 & 34 & ZH161060 & 59 & ZH161068 \\
\hline 10 & ZH161418 & 35 & ZH161054 & 60 & ZH161129 \\
\hline 11 & ZH161276 & 36 & ZH161053 & 61 & ZH161077 \\
\hline 12 & ZH161311 & 37 & ZH161083 & 62 & ZH161066 \\
\hline 13 & ZH161358 & 38 & ZH161120 & 63 & ZH161137 \\
\hline 14 & ZH161303 & 39 & ZH161038 & 64 & ZH161078 \\
\hline 15 & ZH161438 & 40 & ZH161054 & 65 & ZH161114 \\
\hline 16 & ZH161382 & 41 & ZH161051 & 66 & ZH161043 \\
\hline 17 & $\mathrm{ZH} 161434$ & 42 & ZH161054 & 67 & ZH161184 \\
\hline 18 & ZH161377 & 43 & ZH161082 & 68 & ZH161050 \\
\hline 19 & $\mathrm{ZH} 161458$ & 44 & ZH161063 & 69 & ZH161207 \\
\hline 20 & ZH161452 & 45 & ZH161093 & 70 & ZH161089 \\
\hline 21 & ZH161473 & 46 & ZH161071 & 71 & P3502 \\
\hline 22 & ZH161289 & 47 & ZH161210 & 72 & DKC9144 \\
\hline 23 & ZH161471 & 48 & ZH161102 & 73 & NK30 \\
\hline 24 & $\mathrm{ZH} 161285$ & 49 & ZH161398 & 74 & P339 \\
\hline 25 & ZH161135 & 50 & ZH161410 & 75 & Hytech 5106 \\
\hline
\end{tabular}


Study on Applicability of Genotype $\times$ Yield $\times$ Trait $($ GYT) Biplots over Genotype $\times$ Trait $(G T)$ Biplots in Selection of Maize

component analysis (PCA) of standardized data was carried to display the individual trait and yield $\times$ traits relationships. To generate GT and GYT biplots, standardization of twoway table of genotype and trait data was carried out as per procedure given in Yan et al. (2010), Yan and Frégeau-Reid (2018) and Yan et al. (2019). Correlation analysis and construction of GT and GYT biplots were performed through 'metan' version 1.8.1 package (Olivoto and L'ucio, 2020) in RStudio (RStudio, 2020).

\section{RESULTS AND DISCUSSION}

\section{Analysis of variance}

The mean squares of grain yield and its related traits showed the significant variation for the studied maize genotypes and soil moisture within the season (Table 2). This revealed significant progress could be achieved by selection for the improvement of studied traits with desirable performance. Morphological traits had ample amount variation among genotypes and also at genotype-by-environment level indicated as interaction of genotype with soil moisture in the respective season that results substantial differences in the trait expression. Thus, the genetic variability reported in this experiment could be exploited by maize breeders to develop improved cultivars suitable to the particular moisture conditions.

The range and mean values for each trait for 75 maize hybrids under test environments explained the impact of moisture level on the trait's expression (Fig 1). A very few outliers for measured traits were probably the result of inconsistent expressions indicated stress severity and

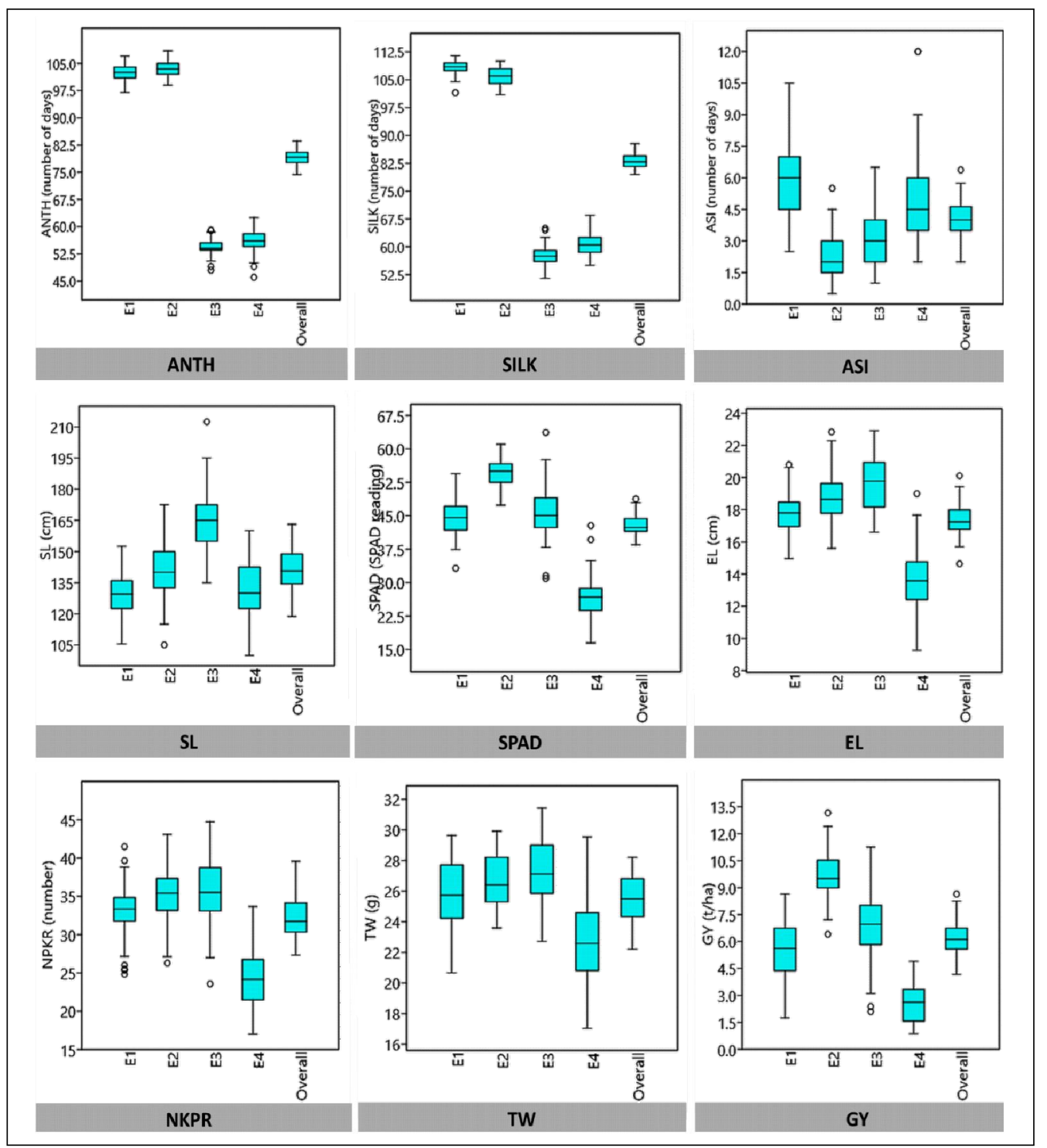

Fig 1: Mean performances of yield and other secondary traits under each test environment.

E1, Rabi 2017-18 under low soil moisture; E2, Rabi 2017-18 under optimal soil moisture; E3, Kharif 2018 under optimal soil moisture; E4, Kharif 2018 under excess soil moisture. 
genotype-by-soil moisture interaction. A large variation in ANTH and SILK in two seasons was observed, that exhibited delayed maturity in winter maize crop due to a prolonged cold period at the early stages of crop growth from seedling to knee-high stage. According to grain yield, hybrids viz, 22 (ZH161289) followed by 30 (ZH161047) and 36 (ZH161053) recorded highest under waterlogging, whereas 36 (ZH161053) followed by 41 (ZH161051) and 9 (ZH161384) showed maximum under drought. Hybrids 22 ( $\mathrm{ZH} 161289)$, 30 ( $\mathrm{ZH} 161047)$ and 49 ( $\mathrm{ZH} 161398)$ had highest yield under optimal whereas 22 (ZH161289), 30 (ZH161047) and 36 ( $\mathrm{ZH} 161053)$ identified as top yielders across test environments.

\section{Correlation studies}

Under drought (E1), GY had significant positive and a fair association with NKPR and SL, whereas a significant and negative correlation with SILK and ASI (Table 3). ANTH had a strong positive association with SILK and a strong negative correlation with ASI and TW. The results revealed that SPAD and EL were not showed significant association with any other trait studied under drought. Under waterlogging (E4), GY showed a significant and positive correlation with SL, EL, NKPR and TW, whereas a significant negative association with ANTH and SILK. A moderate positive correlation observed for ANTH with SILK and a fair relationship with $A S I$ positively. SL showed fair positive association with SPAD and a poor positive association with NKPR. EL recorded a moderate positive correlation with NKPR and TW along with GY, while a poor negative correlation with ANTH. Correlation coefficients estimated for mean values of traits evaluated under both optimal environments (E2 and E3). GY had moderate level of negative correlation with ANTH, SILK and ASI and a positive and significant association with EL, TW, NKPR and SPAD. The flowering traits viz, ANTH, SILK and ASI showed negative and fair association with SPAD, TW, EL and NKPR. Traits such as EL, NKPR and SPAD showed a fair positive

Table 2: Mean sum squares of grain yield and other secondary traits from ANOVA.

\begin{tabular}{|c|c|c|c|c|c|c|c|c|c|c|}
\hline Source of variation & $d f$ & ANTH & SILK & ASI & SL & SPAD & $\mathrm{EL}$ & NKPR & TW & GY \\
\hline GEN & 74 & $28.97^{* * *}$ & $26.24^{\star * *}$ & $5.33^{* \star *}$ & $738.77^{* \star *}$ & $37.08^{*}$ & $7.27^{\star * \star}$ & $50.84^{* * *}$ & $16.66^{* * *}$ & $7.04^{* * *}$ \\
\hline REP & 1 & $14.72^{*}$ & 4.86 & 2.66 & $2934.88^{* *}$ & 0.47 & $62.41^{* * *}$ & 1.55 & 3.37 & $3.92^{*}$ \\
\hline BLK(REP) & 8 & $5.41^{*}$ & 4.25 & 0.61 & 113.43 & 16.81 & 0.71 & 2.83 & 2.00 & $2.97^{*}$ \\
\hline SEA(SMC) & 3 & $114813.08^{* * *}$ & $115281.22^{* * *}$ & $400.42^{* * *}$ & $38331.47^{* * *}$ & $20564.34^{\star \star *}$ & $1008.84^{* * *}$ & $3958.97^{* \star *}$ & $617.21^{* * *}$ & $1296.52^{* * *}$ \\
\hline GEN $\times \operatorname{SEA}(\mathrm{SMC})$ & 222 & $5.00^{* * *}$ & $7.11^{* * *}$ & $4.43^{* * *}$ & $187.97^{\star \star *}$ & $36.34^{*}$ & $3.47^{* * *}$ & $21.67^{* * *}$ & $7.07^{* * *}$ & $2.69^{* * *}$ \\
\hline RESIDUAL & 291 & 3.08 & 3.39 & 2.20 & 118.79 & 28.56 & 0.72 & 3.96 & 1.4 & 1.29 \\
\hline
\end{tabular}

${ }^{* * *}=$ Significant at $0.1 \%(p<0.001),{ }^{* *}=$ Significant at $1 \%(p<0.01),{ }^{*}=$ Significant at $5 \%(p<0.05)$, df: Degrees of freedom; GEN: Genotypes; REP: Replications; BLK: Block; SEA: Season; SMC: Soil moisture condition.

Table 3: Correlation among grain yield and other secondary traits under soil moisture condition.

\begin{tabular}{|c|c|c|c|c|c|c|c|c|c|}
\hline & ANTH & SILK & ASI & SL & SPAD & $\mathrm{EL}$ & NKPR & TW & GY \\
\hline \multicolumn{10}{|c|}{ (a) Correlations under stress (above the diagonal: waterlogging; below the diagonal: drought) } \\
\hline ANTH & 1.00 & $0.77^{* * *}$ & $-0.29^{*}$ & 0.21 & -0.01 & $-0.24^{*}$ & 0.06 & -0.10 & $-0.34^{* *}$ \\
\hline SILK & $0.62^{* \star *}$ & 1.00 & $0.39^{* * *}$ & 0.08 & -0.03 & -0.22 & -0.05 & -0.18 & $-0.45^{\star \star *}$ \\
\hline ASI & $-0.61^{* * *}$ & $0.24^{*}$ & 1.00 & -0.18 & -0.02 & 0.02 & -0.17 & -0.12 & -0.19 \\
\hline $\mathrm{SL}$ & 0.18 & -0.12 & $-0.35^{\star *}$ & 1.00 & $0.23^{*}$ & 0.12 & $0.40^{* * *}$ & -0.04 & $0.26^{*}$ \\
\hline SPAD & -0.11 & -0.10 & 0.03 & 0.04 & 1.00 & 0.02 & $0.24^{*}$ & $-0.23^{* *}$ & -0.01 \\
\hline EL & -0.03 & -0.10 & -0.06 & 0.01 & 0.22 & 1.00 & $0.59^{\star * *}$ & $0.40^{* * *}$ & $0.41^{* * *}$ \\
\hline NKPR & 0.01 & -0.10 & -0.11 & 0.10 & 0.10 & $0.41^{* \star *}$ & 1.00 & 0.19 & $0.43^{* * *}$ \\
\hline TW & $-0.26^{*}$ & $-0.29^{* *}$ & 0.03 & 0.05 & -0.13 & -0.06 & -0.18 & 1.00 & $0.25^{*}$ \\
\hline GY & 0.03 & $-0.32^{* *}$ & $-0.37^{\star *}$ & $0.24^{*}$ & 0.16 & 0.22 & $0.39^{* * *}$ & 0.11 & 1.00 \\
\hline \multicolumn{10}{|c|}{ (b) Correlations under optimal (above the diagonal) and across all the environments (below the diagonal) } \\
\hline ANTH & 1.00 & $0.97^{* * *}$ & $0.49^{* * *}$ & 0.01 & $-0.41^{* * *}$ & $-0.46^{\star * *}$ & $-0.27^{*}$ & $-0.37^{*}$ & $-0.67^{* * *}$ \\
\hline SILK & $0.90^{* * *}$ & 1.00 & $0.69^{* * *}$ & -0.05 & $-0.42^{* * *}$ & $-0.53^{* * *}$ & $-0.35^{\star \star}$ & $-0.44^{* * *}$ & $-0.77^{* \star *}$ \\
\hline ASI & $-0.33^{* *}$ & 0.11 & 1.00 & -0.20 & $-0.27^{*}$ & $-0.50^{* * *}$ & $-0.44^{\star * *}$ & $-0.46^{\star * *}$ & $-0.77^{* * *}$ \\
\hline SL & $0.35^{* *}$ & $0.27^{* *}$ & -0.20 & 1.00 & 0.15 & 0.20 & $0.24^{*}$ & 0.14 & $0.27^{*}$ \\
\hline SPAD & 0.14 & 0.16 & 0.02 & 0.15 & 1.00 & $0.43^{* * *}$ & $0.36^{* *}$ & 0.19 & $0.48^{* * *}$ \\
\hline EL & -0.07 & -0.15 & -0.17 & 0.19 & 0.11 & 1.00 & $0.45^{\star \star *}$ & 0.16 & $0.58^{* * *}$ \\
\hline NKPR & $0.25^{\star *}$ & 0.13 & $-0.31^{\star *}$ & $0.45^{\star *}$ & $0.31^{\star *}$ & $0.58^{* * *}$ & 1.00 & 0.22 & $0.51^{* * *}$ \\
\hline TW & $-0.31^{* *}$ & $-0.34^{* *}$ & -0.04 & -0.15 & $-0.29^{* *}$ & 0.06 & $-0.31^{* *}$ & 1.00 & $0.57^{* * *}$ \\
\hline GY & $-0.24^{*}$ & $-0.35^{\star *}$ & -0.20 & $0.30^{* *}$ & -0.06 & $0.26^{*}$ & $0.27^{*}$ & 0.15 & 1.00 \\
\hline
\end{tabular}

Note: ${ }^{* * *},{ }^{* *}$ and ${ }^{*}$ Significant at $p$-value $0.001,0.01$ and 0.05 respectively. 
Study on Applicability of Genotype $\times$ Yield $\times$ Trait $($ GYT) Biplots over Genotype $\times$ Trait $(G T)$ Biplots in Selection of Maize

relation with each other under the optimal soil moisture environment. GY had a poor positive correlation with NKPR, EL and SL whereas fair to poor negative correlation with ANTH and SILK across all the test environments including low, optimal and excess soil moisture.

A significant negative association recorded between grain yield and flowering traits (ANTH and SILK) under all the moisture conditions, while ASI had significant negative correlation under drought. It explained the importance of ASI in development of drought tolerant maize cultivars. The longer gap between anthesis and silking resulted in failure of proper seed set due to poor availability of viable pollen for late emerged female flowers. Campbell et al. (2014) reported shorter ASI is genetically correlated with increased drought tolerance by studying the effect of water availability on flowering behavior under moisture regimes. Longer ASI values are owing to the delay of silk extrusion, premature lodging and reduced rates of net photosynthesis arising from oxidative damage to chloroplasts (Nelimor et al. 2020). The relationship between grain yield and ASI in our study was in agreement with previous reports on maize under

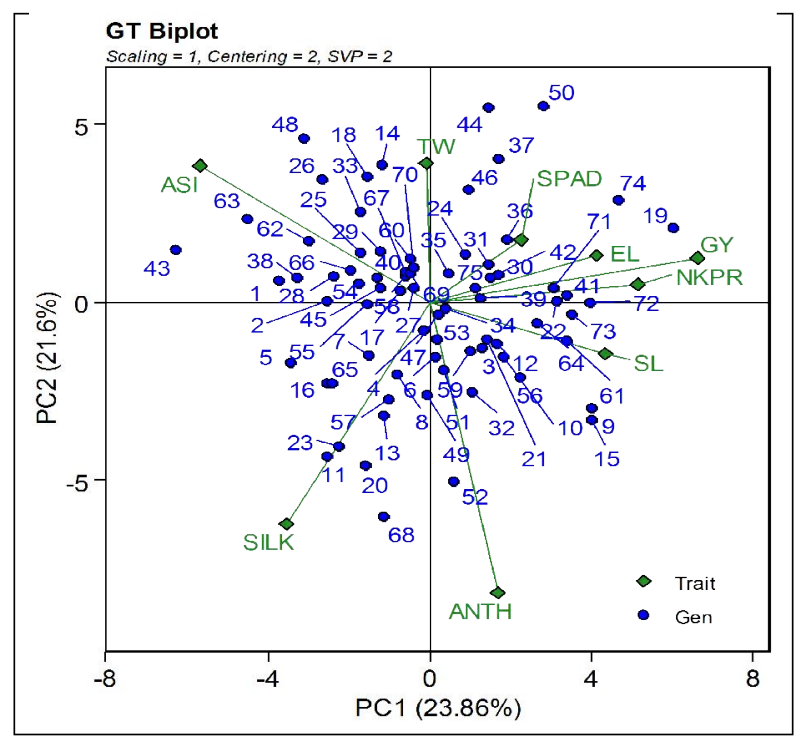

(A)

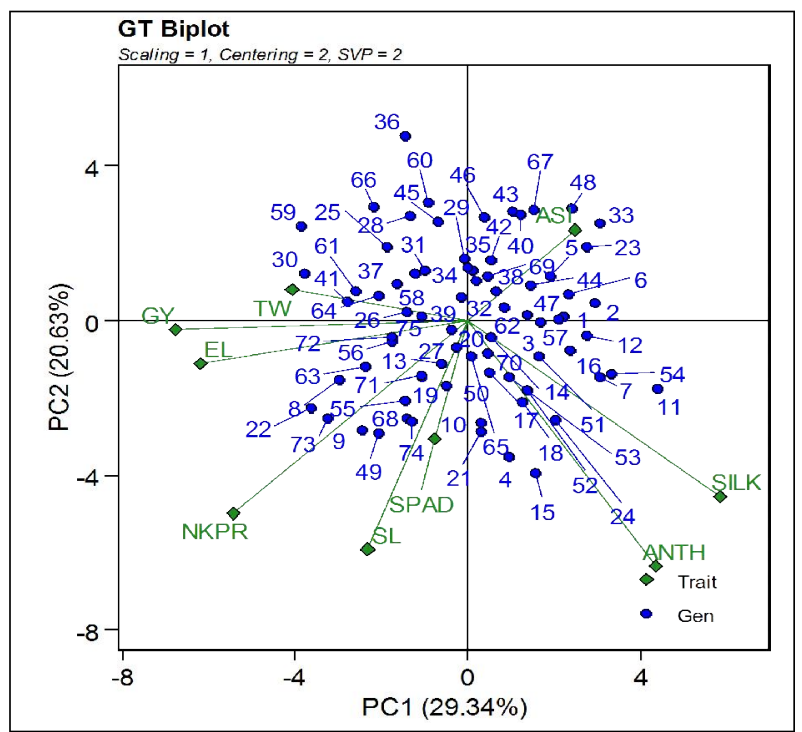

(B)

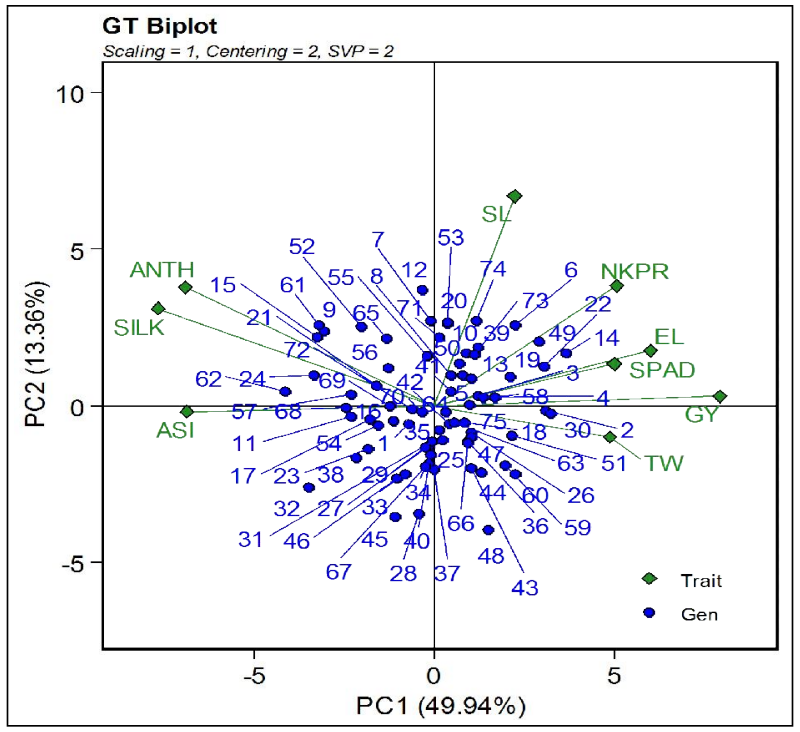

(C)

Fig 2: Genotype by trait (GT) biplot illustrating the relationship between PC1 and PC2 for 75 genotypes and nine traits under (a) low soil moisture (b) excess soil moisture (c) optimal soil moisture.

PC1 and PC2, principal components 1and 2 respectively; Scaling = 1, Centering $=2$ and SVP = 2 represented the biplot was based on singular value decomposition of trait-standardized data and trait-focused singular value partition. Goodness of fit for biplot (a) $45.46 \%$, (b) $49.97 \%$ and (c) $63.30 \%$. 
drought (Edmeades and Daynard, 1979) and under waterlogging conditions (Zaidi et al. 2007). Direct selection of characters like SL, EL and NKPR would results in promising yields under varied moisture conditions. The strong association between phenological traits and grain yield under water deficit conditions than under optimal moisture conditions was reported by Sah et al. (2020).

\section{GT and GYT biplots}

According to GT biplots, the first two PCs explained $45.46 \%$ of total variability under LSM, whereas $49.97 \%$ and $63.3 \%$ under ESM and OSM conditions, respectively (Fig 2a-c). In

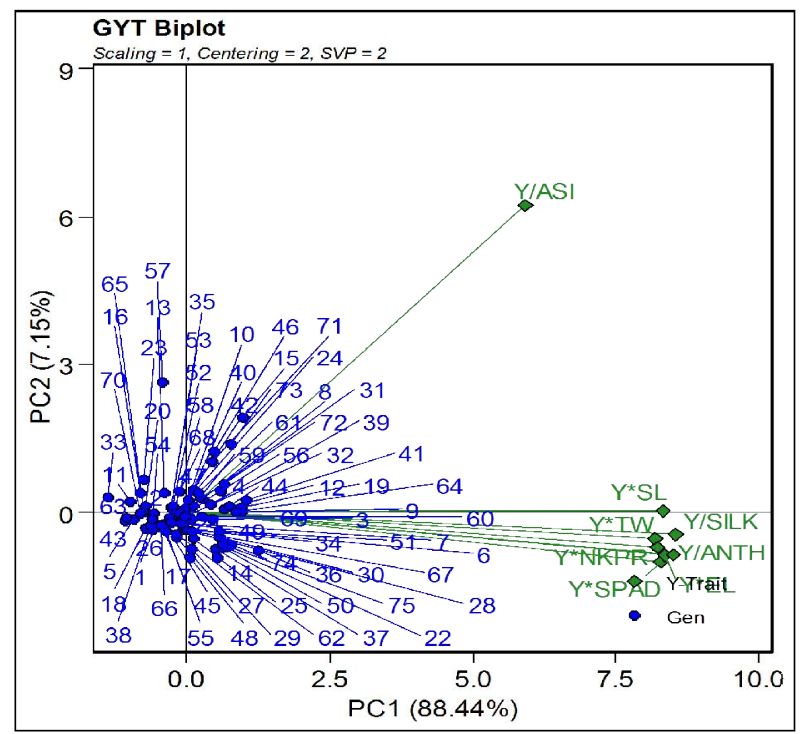

(A) case of GYT biplots, first two PCs contributed around 95\% under LSM and ESM, whereas 90\% under OSM (Fig 3a-c). For example, biplot (Fig 2a) constructed for performance of genotype under drought environment showed that GY was positively correlated with EL, SL, NK, PR and negatively with ASI and SILK. Almost zero correlation was found for GY with TW and ANTH. Also the biplot depicted the cultivars viz, 19 (ZH161458), 72 (DKC9144), 41 (ZH161051), 39 (ZH161038), 73 (NK30) and 22 (ZH161289) etc. were recorded higher values for traits viz, NKPR, SL, EL and GY.

The accuracy of biplots was based on goodness of fit that generally indicated by the sum of PCs involved in the

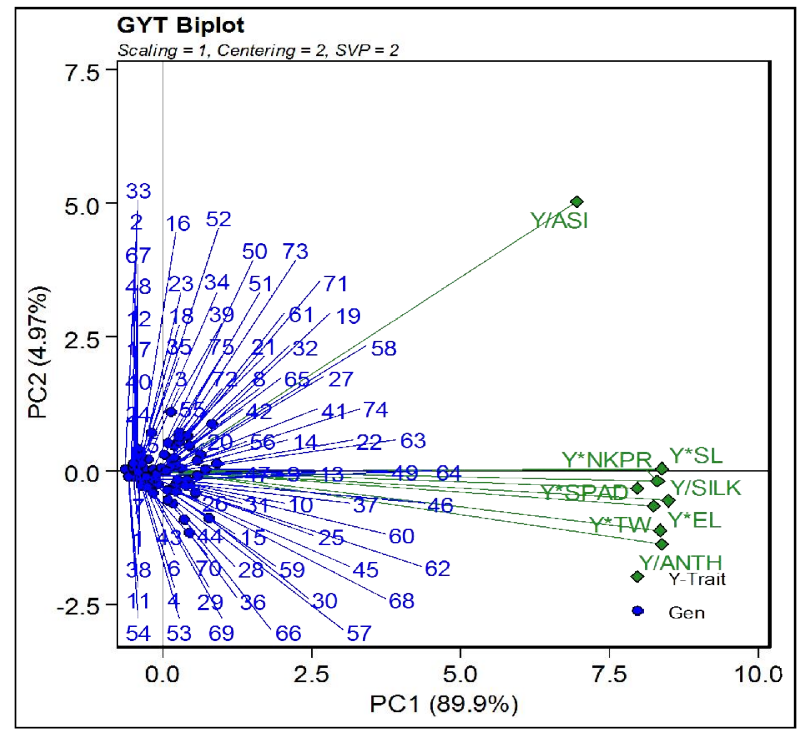

(B)

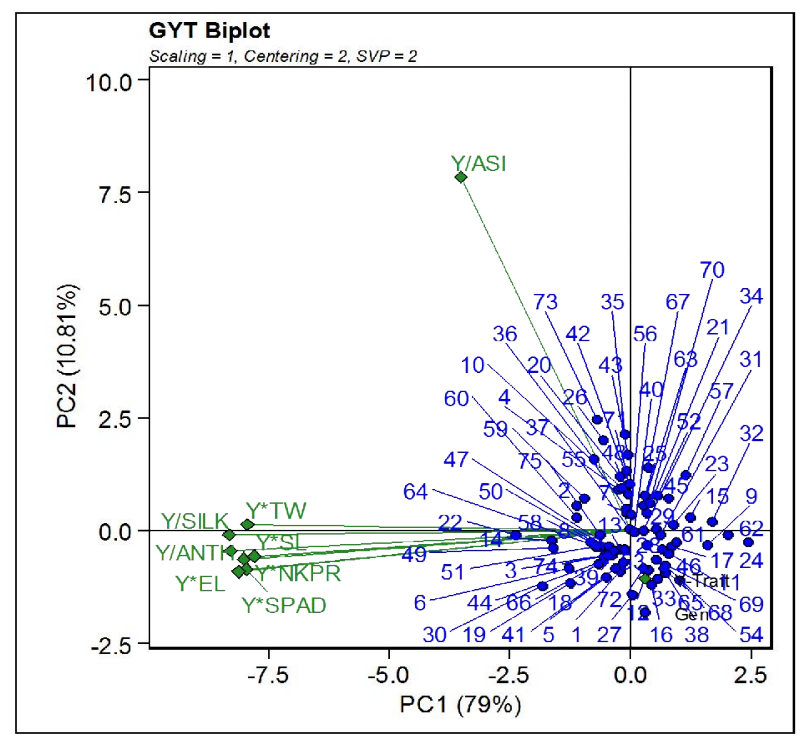

(C)

Fig 3: Genotype by yield*trait (GYT) biplot illustrating the relationship between PC1 and PC2 for 75 genotypes and nine traits under (a) low soil moisture (b)excess soil moisture (c) optimal soil moisture.

PC1 and PC2, principal components 1 and 2 respectively; Scaling = 1, Centering $=2$, and SVP $=2$ represented the biplot was based on singular value decomposition of trait-standardized data and trait-focused singular value partition. Goodness of fit for biplot (a) $95.59 \%$, (b) $93.87 \%$ and (c) $89.81 \%$. 
Study on Applicability of Genotype $\times$ Yield $\times$ Trait (GYT) Biplots over Genotype $\times$ Trait (GT) Biplots in Selection of Maize

biplot construction. The goodness of fit for the GT biplots constructed under all the studied moisture conditions were almost poor due to fair to moderate correlations among the traits. To accomplish the problem of weak associations, GYT technique was advisable to perform the precise selection of genotypes under studied environments. However, each trait combined with yield is more acceptable than yield per se alone or each trait alone in case of multiple trait evaluation. Correlations among yield-trait combinations are obviously greater than the trait-associations as the result of presence of yield as a common component. This could be observed by the presence of acute angles between yield-trait vectors in the GYT biplots (Fig 3a-c). The studied hybrids can be depicted graphically on GYT biplots based on these strong yield-trait correlation values. The GYT biplots showed the enhanced goodness of fit than the GT biplots. Around $90-95 \%$ goodness of fit that explained very good adequacy in biplot construction was recorded for all the soil moisture environments. Further, ranking and selection of genotypes could be proceeded with respect to desirable yield-trait combinations by plotting polygon view and mean-stability plot with similar interpretations of GGE biplots (Yan and Tinker, 2005).

\section{CONCLUSION}

The measured traits had ample amount variation among genotypes and also at genotype-by-environment level indicated as interaction of genotype with soil moisture in the respective season that results substantial differences in the trait expression. To overcome the problem of weak associations in GT biplots under stress environments, GYT technique was desirable to perform the precise selection and genotype ranking pattern of genotypes under test environments where the grain yield is targeted.

\section{ACKNOWLEDGEMENT}

The authors are thankful to Banaras Hindu University, Varanasi and CIMMYT, Hyderabad for facilitating required needs as well as constant support and financial assistance during the entire research work.

\section{Conflict of interest}

The authors declare no conflict of interest.

\section{REFERENCES}

Al-Naggar, A.M.M., Shafik, M.M., Musa, R.Y.M., Younis, A.S.M. and Anany, A.H. (2020). Genetic variability of maize hybrids and populations and interrelationships among grain yield and its related traits under drought and low $\mathrm{N}$ using multivariate analysis. Asian Journal of Biochemistry. Genetics and Molecular Biology. 4(2): 26-44.

Bolaños, J. and Edmeades, G.O. (1993). Eight cycles of selection for drought tolerance in lowland tropical maize. I. Responses in grain yield, biomass and radiation utilization. Field Crops Research. 31: 233-252.

Campbell, L., Luo, J. and Mercer, K. (2014). Effect of water availability and genetic diversity on flowering phenology, synchrony and reproductive investment in maize. Maydica. 59: 283-289.
Edmeades, G.O. and Daynard, T.B. (1979). The development of plant-to-plant variability in maize at different planting densities. Canadian Journal of Plant Sciences. 59: 561576.

Nelimor, C., Badu-Apraku, B., Tetteh, A.Y., Garcia-Oliveira, A.L. and N'guetta, A.S. (2020). Assessing the potential of extra-early maturing landraces for improving tolerance to drought, heat, and both combined stresses in maize. Agronomy. 10: 318.

Olivoto, T. and L'ucio, A.D. (2020). Metan: An R package for multienvironment trial analysis. bioRxiv. https://doi.org/ 10.1101/2020.01.14.906750.

Rstudio. (2020). RStudio: Integrated Development for R. RStudio. Inc., Boston, MA.

Sah, R.P., Chakraborty, M., Prasad, K., Pandit, M., Tudu, V.K., Chakravarty, M.K., Narayan, S.C., Rana, M. and Moharana, D. (2020). Impact of water deficit stress in maize: Phenology and yield components. Scientific Reports. 10: 2944.

SAS Institute. (2009). The SAS System for Windows. Version 9.2. Cary, CN: SAS Institute.

Sofi, P.A., Saba, I., Ara, A. and Rehman, K. (2021). Comparative efficiency of $\mathrm{GY}^{*} \mathrm{~T}$ approach over GT approach in genotypic selection in multiple trait evaluations: case study of common bean (Phaseolus vulgaris) grown under temperate himalayan conditions. Agricultural Research. $1-9$.

Yan, W. and Frégeau-Reid, J. (2018). Genotype by yield*trait (GYT) biplot: A novel approach for genotype selection based on multiple traits. Scientific Reports. 8: 8242.

Yan, W., Frégeau-Reid, J., Mountain, N. and Kobler, J. (2019). Genotype and management evaluation based on genotype by yield*trait (GYT) analysis. Crop Breeding, Genetics and Genomics.1: e190002.

Yan, W., Frégeau-Reid, J., Pageau, D., Martin, R., Mitchell-Fetch, J., Etienne, M., et al. (2010). Identifying essential test locations for oat breeding in Eastern Canada. Crop Science. 50(2): 504-515.

Zaidi, P.H., Maniselvan, P., Sultana, R., Yadav, M., Singh, R.P., Singh, S.B., Dass, S. and Srinivasan, G. (2007). Importance of secondary traits in improvement of maize (Zea mays L.) for enhancing tolerance to excessive soil moisture stress. Cereal Research Communications. 35(3): 1427-1435.

Zaidi, P.H., Mehrajuddin, Jat, M.L., Pixley, K., Singh, R.P. and Dass, S. (2008). Resilient maize for improved and stable productivity of rain-fed environment of South and SouthEast Asia. Maize for Asia: Emerging Trends and Technologies. Proc. $10^{\text {th }}$ Asian Regional Maize Workshop. 20-23 October, 2008, Makassar, Indonesia.

Zaidi, P.H., Vinayan, M.T. and Seetharam, K. (2016). Phenotyping for abiotic stress tolerance in maize: Water logging stress. A field manual. CIMMYT: Hyderabad, India.

Zaman-Allah, M., Zaidi, P.H., Trachsel, S., Cairns, J.E., Vinayan, M.T. and Seetharam, K. (2016). Phenotyping for abiotic stress tolerance in maize: Drought stress. A field manual. CIMMYT, Mexico. 